\title{
$\mathrm{CiSj}$
}

\section{EXPERIMENTAL CHARACTERIZATION OF RFID SYSTEMS FOR PROCESS CONTROL IN INDUSTRIAL MARBLE MACHINES}

\author{
Sergio Saponara, Fabrizio lacopetti, Luca Fanucci, Bruno Neri \\ Department of Information Engineering, University of Pisa, Via Caruso, 16, I-56122, ITALY, \\ \{sergio.saponara, fabrizio.iacopetti, luca.fanucci, b.neri\}@iet.unipi.it, www.iet.unipi.it
}

\begin{abstract}
The paper presents an experimental characterization of wireless systems, specifically RFID technologies, applied to polishing/cutting process control in the marble industry. The application of RFID systems has the final aim of allowing the automatic and contact-less detection of the presence of a marble slab in different points of a marble machine, outside and/or inside, trying to overcome some limitations of the currently used proximity detectors. Slab detection is needed for the process control in order to properly activate the abrasive or cutting heads of the machine. Four RFID systems at $125 \mathrm{kHz}, 13.56 \mathrm{MHz}, 868 \mathrm{MHz}$ and $2.45 \mathrm{GHz}$ have been tested in different set-ups representative of those found in marble machines. Starting from commercially available tags, readers and antennas, ad-hoc developed or customized hardware and/or software have been used for the experimental test campaign, that has been carried out also considering dirty and wet working environments representative of those found in real applications.
\end{abstract}

Keywords: RFID (Radio Frequency IDentification), Contact-less industrial measurements, Wireless systems, Marble industry, Process control.

\section{INTRODUCTION}

Several different industrial processes are nowadays used in order to transform a stone block coming from quarries into a finished product, as an example a tile, a sculpture, stone powder. In the field of slab-shaped products (e.g. tiles) marble blocks coming from quarries are firstly sawn by a gangsaw [1] into marble slabs with a resulting irregular shape and rough surfaces; slabs are afterwards polished in a polishing machine [2] and later cut into smaller slabs by means of cutting machines, possibly subjected to other processes and finally becoming end products. This paper deals in particular with the use of wireless systems, specifically RFID-based techniques, to improve the automation of industrial machines for marble slabs polishing, but the discussion is still applicable to other machines for stone slab working (cutting, waxing, etc.). The use of wireless techniques in industrial applications is an interesting and emerging application trend, aiming at reducing cabling and installation costs, at avoiding the danger of cables and connectors failure especially in moving parts of industrial machines. Recently several works have been proposed in literature [3] mainly addressing wireless systems for industrial communication or in case of RFID techniques, for positioning and logistics. On the contrary this work exploits RFID wireless technologies for proximity sensing to improve the industrial process control in marble machines. This tentative, new with respect to the state of the art, is supported by experimental campaigns that consider working environments representative of those found in real industrial applications.

Fig. 1 shows the schematic diagram of a typical industrial marble machine for slab working. Marble machines are typically made up of consecutive working heads (up to a few dozens) under which the marble slab, initially brought to the machine by means of a roller system, is transported by a plastic conveyer belt [2]. A marble slab is typically sized 2 $\mathrm{m} \times 3 \mathrm{~m}$ and has a thickness of some $\mathrm{cm}$. The typical conveyer belt speed amounts to few $\mathrm{cm} / \mathrm{s}$. As an example, the polishing machine in [2] has a total length of $13.5 \mathrm{~m}$ and up to 18 abrasive heads. The working environment inside a marble machine is dirty, due to mud, marble and abrasive residuals, and wet, due to a water level of few $\mathrm{cm}$ needed for heads cooling, elimination of residuals, easing of the conveyer belt sliding on the machine metallic plane. The above issues add up to the other classic problems of wireless systems in industrial scenarios, such as multi-paths and interference from other electromagnetic sources.

Currently, the process control for marble slab working is based on the following scheme: mechanical or optical sensors detect the presence of 


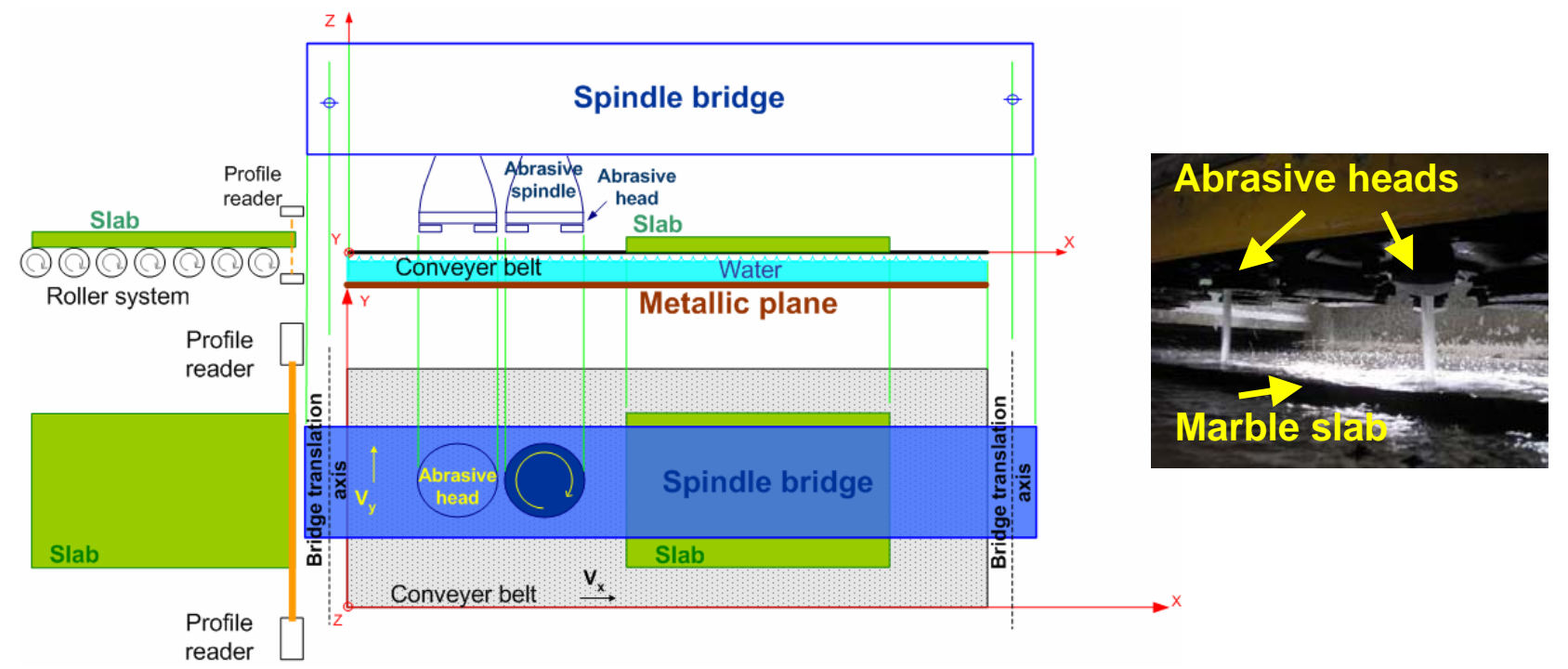

Fig. 1 - Schematic diagram of a marble machine and a snapshot of the abrasive heads over a marble slab

the marble slab on the conveyer belt at the entrance of the industrial machine. Such information is used by the PLC (Programmable Logic Controller) controlling the entire machine, including the conveyer belt speed, as the reference time to drive down each cutting/abrasive head when the marble slab is under it, and, when the slab is passing the working head, to drive up the head in the initial position. The above mentioned feed-forward control rule is based on the assumptions that the position and speed of the conveyer belt, the position of the marble slab on the belt and therefore the relative positions of the heads are constantly known. If one of such assumptions is not verified then the marble slab is not present when the head is driven down; the latter reaches and damages the conveyer belt which must be repaired or replaced causing a long stop of the machine and of the industrial process. During the long path inside the machine, the slab may move on the conveyer belt due to the working itself or due to break under the working mechanical stress. Hence a feedback detection signal should be provided to the heads control system about the real presence of the marble slab under the head inside the machine.

The rest of this work, which extends the IEEE IDAACS 2009 conference paper [4], is organized as follows. Section 2 reviews state-of-art solutions for contact and contact-less detection in industrial applications, particularly in marble industry. Section 3 introduces the 4 RFID systems analyzed and tested in the present work, each one based on a different RFID technology. Sections 4, 5, 6 and 7 present the applications and results concerning the test of the 4 mentioned RFID technologies applied to the case of slab detection in conditions representative of a real industrial working scenario. A comparison among the different analyzed solutions and conclusions on contact-less proximity detection for marble industry are reported in Section 8.

\section{SENSORS FOR PROCESS CONTROL IN MARBLE INDUSTRY}

In addition to the missing feedback to the heads control system inside the machine, state of the art sensors for slab detection outside the machine have some problems still to overcome: mechanical sensors suffer of rapid deterioration due to the continuous contact with the slab while optical sensors [5] need frequent cleaning and re-calibration due to the dirty working environment (see Fig. 2).

Due to the wet and dirty working conditions, and to the non-homogeneous and non-constant environmental physical properties, also other contact-less sensors proposed or potentially suitable for marble machines, based on LASER or vision systems, or ultrasonic waves $[6,7,8]$ were not suitable for successful industrial applications.

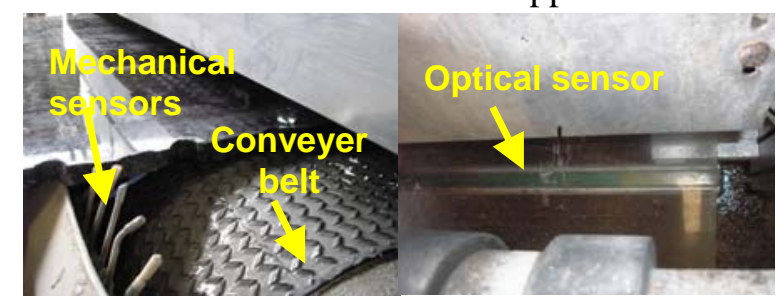

Fig. 2 - Mechanical sensors and optical sensors at the machine entrance stage

Capacitive sensors for marble [9] and more complex ultra sound- or georadar-based systems [6, $10,11]$ have been studied in literature. However their target is the fine-grain analysis of the porosity and defects of stone materials (e.g. based on dielectric permittivity variations) in a controlled working environment (dry, clean, with still stone samples) rather than the run-time detection of a marble slab in an industrial machine. In [12] we have presented preliminary results from experimental tests exploiting capacitive sensing for 
on/off marble slab detection at the entrance and inside the marble machine, by means of two main approaches: (a) using a capacitor realized by means of a metallic plate suspended over the machine metallic plate, see Fig. 3; (b) using a newly-designed version of the surface capacitor proposed in [9].

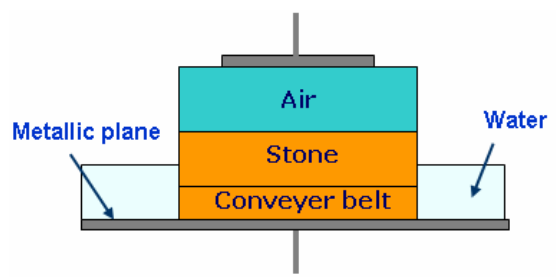

Fig. 3 - A multi dielectric layer (air/stone/plastic) capacitive sensor inside the marble machine

Proximity capacitive sensors have also been proposed by semiconductor industry in [13] but targeting small distances mainly for touch sensing applications. Also the techniques proposed in $[6,7$, $8,11,14,15]$ aim at classification and fine-grain analysis of the texture and surface of stone slabs in a static and controlled environment, with conditions different from those found inside a marble machine. Moreover, for marble machines a simpler on/off detection is required, but with the possibility to be performed in real-time, with higher robustness, to be easily integrated with the machine controlling system, having low maintenance costs. Finally, the computational power needed to implement computing techniques based on wavelets, Gabor filters or neural networks as in [7, 8, 14] is not compatible with the utilization of the PLC devices commonly used in marble machines controlling systems, above all if such techniques must be applied in several points inside the machine.

To address some of the issues of state of the art slab detection, this paper presents the experimental characterization of 4 different RFID systems used to detect the presence of the marble slab outside and/or inside the marble machine. Our project aims at a sensing scheme being contact-less, nearly maintenance free, operating in real-time, and robust to harsh environment conditions.

At the state of the art the application of RFID technologies has been analyzed and implemented for logistics (for which commercial solutions are available, as an example in the marble industry [16, 17] using $13.56 \mathrm{MHz}$ passive tags and handheld RFID reader), for the management of production $[18,19]$, and even for localization [20], but less for industrial machine process control and sensing. The target of this work is highlighting the advantages and limits of RFID systems applied to detection tasks, mainly in the marble industry process control, and suggesting which technologies are most suitable and how they can be used.

\section{RFID FOR PROCESS CONTROL IN MARBLE MACHINES}

RFID is a means of identifying, but also tracking and detecting, an item using radio frequency communication, which takes place between a transmitter, usually called "reader", and a transponder (silicon chip connected to an antenna), usually called "tag". The physical coupling is based on magnetic or electromagnetic fields. Tags can either be passive, i.e. powered by the reader field, semi-passive or active, i.e. powered by a battery; in this last case the transmitter is usually the tag itself.

For marble machines the effects on reader-tag communication depend on various parameters: the composition and shape of the stone slab, the operating frequency and power levels of the RFID system, the radiation pattern of the antennas, the distance between tag and reader and their relative orientation, the working environment (presence of water or dust or a mixture of both, presence of metallic planes in the machine, composition of the conveyer belt). Hence an experimental test campaign on real case studies using different RFID systems is required. Such experimental campaign, missing in literature, is the main objective of this work.

Since marble machines are not produced in large numbers, so justifying the development of ASICs, in our experiments we implemented 4 RFID systems starting from commercially available tags and readers, and customizing the hardware components and/or the relevant software for proper configuration of the experimental set up and for test results acquisition and processing. Due to the poor availability of experimental data in literature, we have investigated the application of RFID systems ranging from the Low Frequencies (LF) to the Ultra High Frequencies (UHF) bands. The four considered RFID systems are shown in Fig. 4: the Low Frequency RFID system of Fig. 4.a [21], using passive tags and a communication frequency of 125 $\mathrm{kHz}$; the High Frequency (HF) system of Fig. 4.c at 13.56 MHz using passive tags [22]; two Ultra High Frequency systems, at $868 \mathrm{MHz}$ [23], see Fig. 4.b, using passive tags and at $2.45 \mathrm{GHz}$ [24], see Fig. 4.d, using active tags.

The following experiments and related hardware and/or software customizations of the systems in Fig. 4 have been realized: (i) measurement and comparison with a given threshold of the amplitude of the signal modulated by the tag and decoded by the reader; (ii) proper setting, through the control software, of the power radiated by the reader antenna; (iii) detection and comparison with a given threshold of the packet reception error rate in the tag-reader communication. The above solutions are alternative each other and the most suited depends 


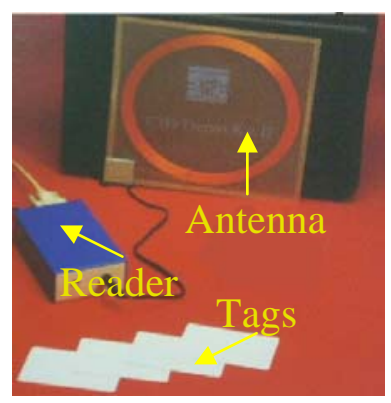

a) $\mathrm{LF} 125 \mathrm{kHz}$

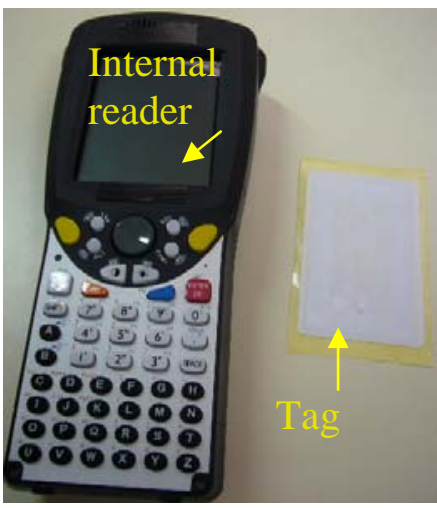

c) $\mathrm{HF} 13.56 \mathrm{MHz}$

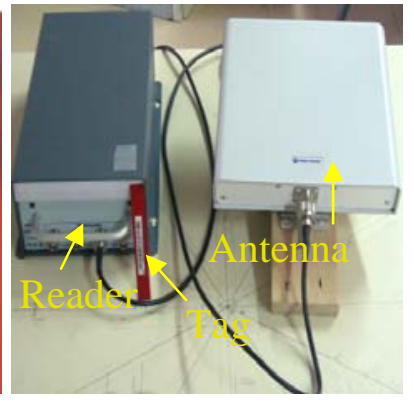

b) UHF $868 \mathrm{MHz}$

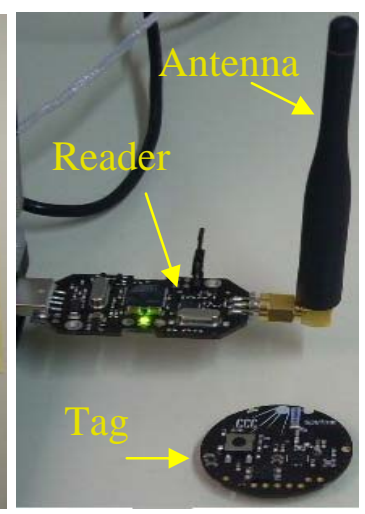

d) UHF $2.45 \mathrm{GHz}$
Fig. 4 - Considered RFID systems (reader, antenna and tag)

on the possibility of configuration offered by RFID components. As an example in the considered systems [23] and [24] the reader power level may be configured. The software of [24] allows processing of communication data to determine the packet error rate. For the test campaign sixteen stone samples, different in size and shape (typically rectangular with the larger sizes in the order of some tens $\mathrm{cm}$ and height of up to a few $\mathrm{cm}$ ) and representative of the possible materials processed in marble industry (e.g. onyx, marble, granite, ..) have been considered. In Fig. 5 six of the sixteen stone samples are shown.

\section{EXPERIMENTAL ANALYSIS OF LF RFID SYSTEMS}

The $125 \mathrm{kHz}$ LF RFID system has been firstly characterized in a test set up reproducing the environment inside the marble machine; as sketched in Fig. 6 the passive tags have been embedded in fixed positions in the conveyer belt which is made of plastic/rubber and is transparent to LF (the scheme in Fig. 6 realizes a "smart conveyer belt"). The aim of the first test has been the determination of the maximum distances in the $3 \mathrm{D}$ space where the tags are detected by the reader without the interposition of the stone samples. In this test the observed output is the digital output of the RFID LF reader, see Fig. 7 , sent to a PC connected to the reader and shown by means of an application providing on/off detection information (plus the code of the tag in case of
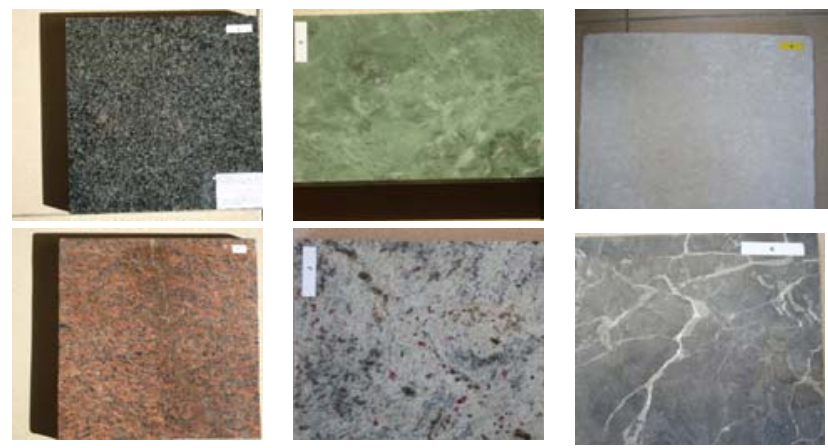

Fig. 5 - Six of the 16 marble slabs used for the tests

detection). Reader functionalities are provided by an embedded Intel 80386 processor. The LF reader, using a coil antenna with a diameter of about $20 \mathrm{~cm}$ (see Fig 4.a), communicates with passive tags of size $8 \mathrm{~cm} \times 5 \mathrm{~cm}$ through inductive coupling. The output power of the reader is $100 \mathrm{~mW}$. Fig. 7 also shows the waveform of the signal on reader coil, highlighting the carrier and its modulation (communication data).

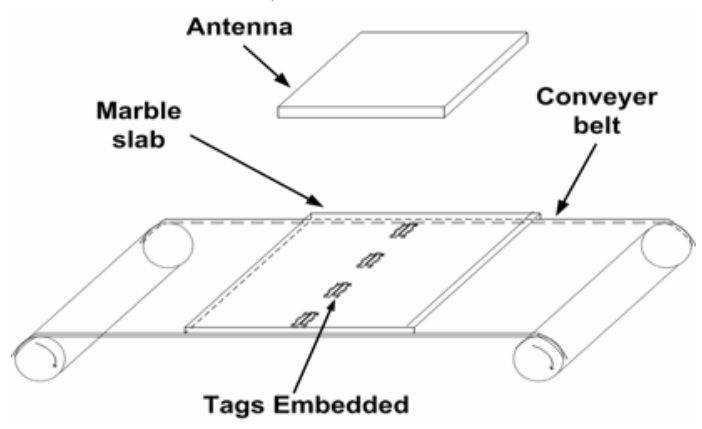

Fig. 6 - Test configuration with tags embedded in the conveyer belt

The experimental results, reported in Fig. 8, show that the tags can be detected up to a distance of roughly $20 \mathrm{~cm}$ in the $3 \mathrm{D}$ space. The experiment has been repeated with all the different stone samples interposed between the tags and the reader coil antenna, with and without the presence of a water layer of some $\mathrm{cm}$ and with and without the presence of marble dust and mud. The obtained results are substantially the same of those reported in Fig. 8.

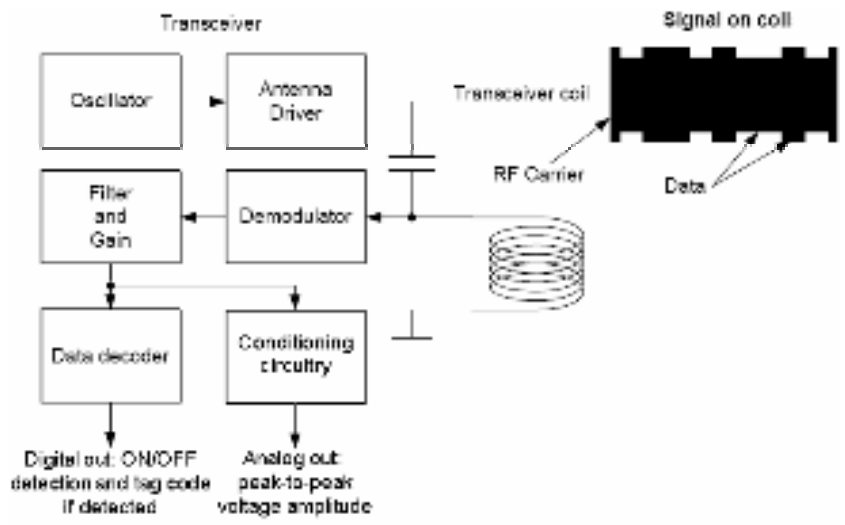

Fig. 7 - Architecture of the reader, LF RFID system 


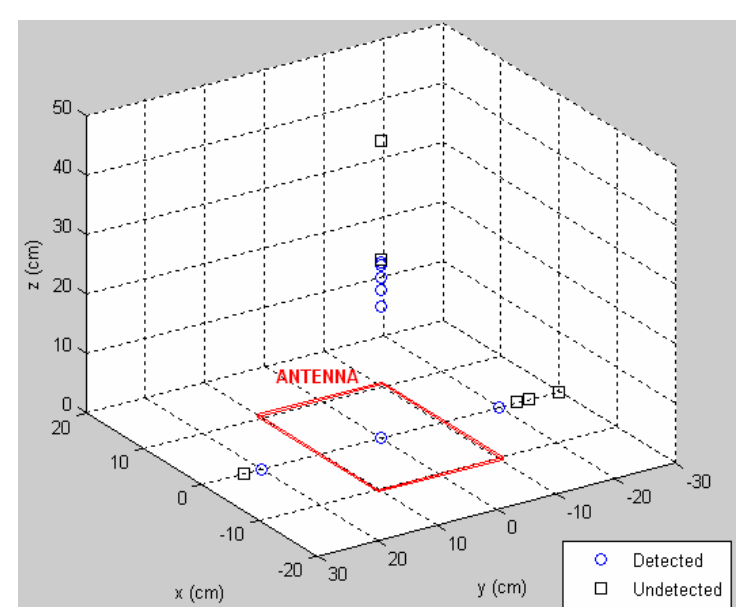

Fig. 8 - Experimental points of detection, LF RFID system.

Experimental results prove that stones, as expected at the test frequency, water and mud are almost transparent to LF radiations. Finally we repeated all the above tests analyzing the analog decoded signal in the LF reader, see Fig. 7, considering a fixed distance of $10 \mathrm{~cm}$ (compliant with the use of the system inside the machine) between the nearest tag and the reader coil antenna. The analyzed signal is obtained through a custom circuit we added to the reader circuitry in order to filter and measure the amplitude of the decoded signal (envelope of the received signal). Results of these tests are showed in Fig. 9. Each reported measurement is generated by averaging ten measurements of the peak-to-peak signal amplitude, with and without interposition of the stone slab between the tag and the reader. The peak-to-peak voltage level measured in absence of stone samples resulted to be $120 \mathrm{mV}$. Repeating the tests with the stones interposed (more than 16 different stone samples labeled with letters from A to P in Fig. 9, with/without water or mud or marble dust), the revealed signal ranged between $115 \mathrm{mV}$ and 125 $\mathrm{mV}$ with small differences (within $\pm 4 \%$ ) vs. the 120 $\mathrm{mV}$ reference (see Fig. 9). Since the measured value slightly depends on the specific type of stone the above detecting technique could possibly be used for industrial applications involving a specific stone type but not in a machinery where the type of stone samples varies from slab to slab.

To solve this issue for the LF RFID system a different detecting strategy has been adopted: instead of embedding the tags in the conveyer belt as in Fig. 6, the tag has been applied on the side surface of each marble slab through a fast dry resin as in [16]. The tag is not placed on the top surface of the marble slab in order to avoid any damage by the polishing abrasive heads. The tag has in this case the function of confirming the presence of the slab on the conveyer belt in the position expected by the PLC. This configuration allows the detection, even in presence of water and dirt, of the tag and of the corresponding marble slab when the tag is passing in the area covered by the reader antenna. For the considered LF system the antenna-tag distance should be within $15 \mathrm{~cm}$. Such distance allows the application both outside and inside the machine.

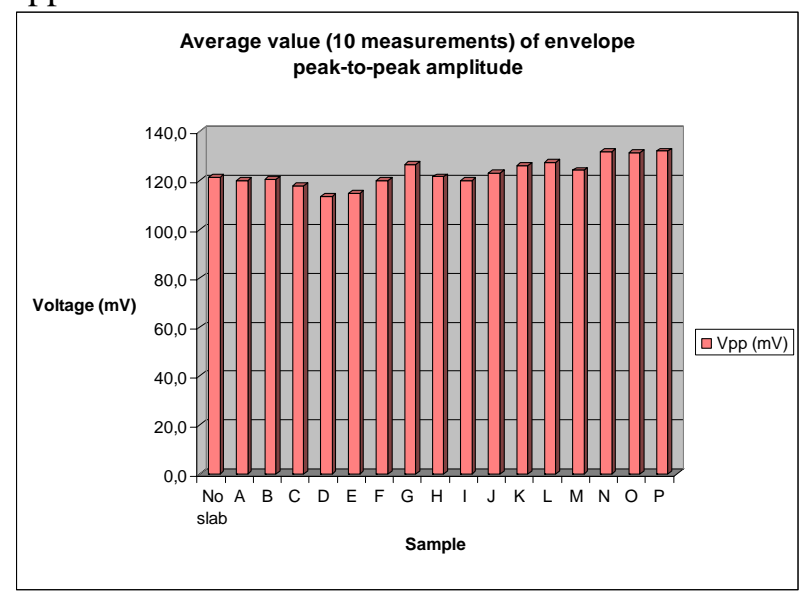

Fig. 9 - Peak-to-peak amplitude of the decoded signal picked up on the reader by a custom circuitry

\section{EXPERIMENTAL ANALYSIS OF HF RFID SYSTEMS}

For the RFID HF system in Fig. 4.c, communicating through magnetic coupling with passive tags at $13.56 \mathrm{MHz}$, by means of a Windows CE application on the handheld device we implemented similar tests and obtained similar results as in the case of the RFID LF system. Fig. 10 shows the experimental results concerning the detection limit points in the 3D space without any stone sample interposition. The only difference with the LF system is that the HF system is based on a handheld battery-powered device [22] with an internal tag reader and an internal antenna. Due to the limited radiated power, in the range of tens $\mathrm{mW}$

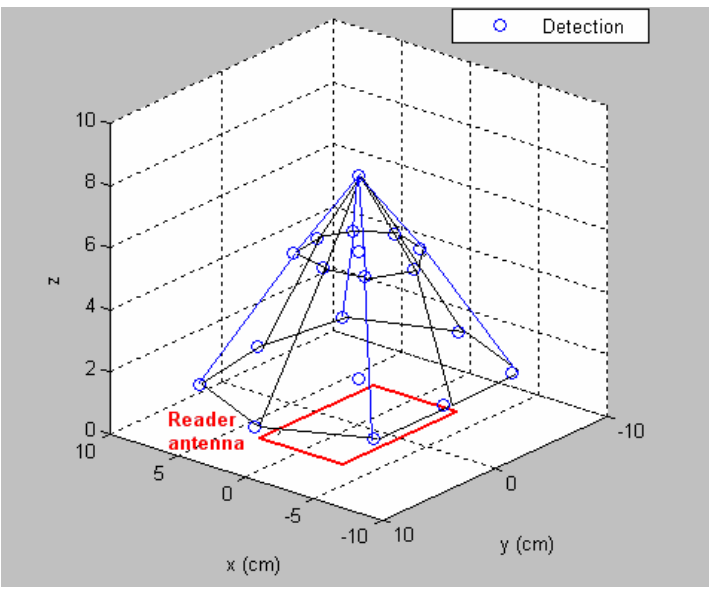

Fig. 10 - Experimental surface of maximum detection distance, HF RFID system

(typical of HF RFID readers for handheld 
applications [25, 26]), the maximum reachable distance is below $8 \mathrm{~cm}$. Using an HF RFID reader, not battery-powered, a higher power level could be irradiated and hence we expect the achievement of performances similar to those of the LF system concerning the maximum distance of tag reading.

\section{EXPERIMENTAL ANALYSIS OF UHF RFID SYSTEMS}

For the RFID UHF system, shown in Fig. 4.b and working at $868 \mathrm{MHz}$, we have firstly repeated the characterization with the test set up in Fig. 6 reproducing the marble machine working environment, embedding the passive tags in fixed positions in or below the conveyer belt. The aim of the first test was the determination of the maximum distances in the 3D space at which the tags are detected by the UHF reader for a defined tagantenna relative orientation, without interposition of stone samples, at different radiated power levels. Experimental results are reported in Fig. 11 for the case example of the reader radiating a $100 \mathrm{~mW}$ power. At UHF frequencies the coupling between RFID reader and tags is electromagnetic.

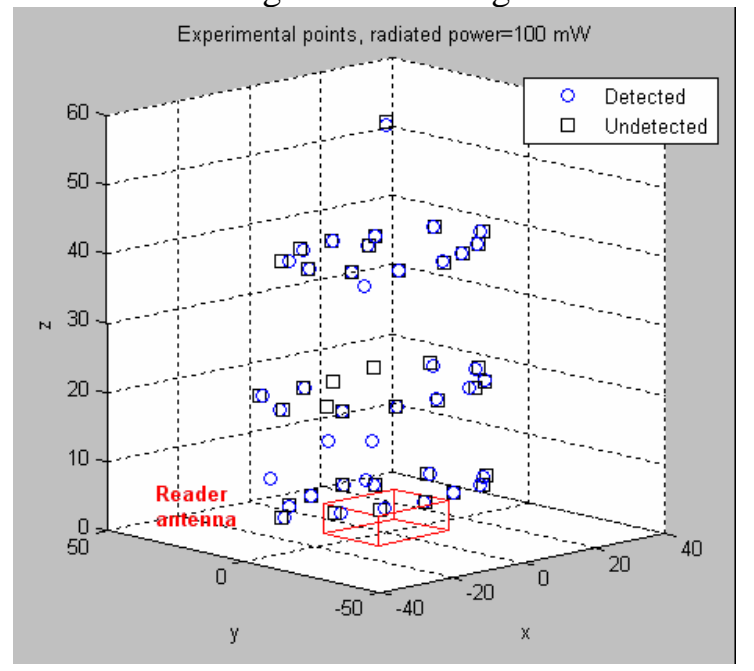

Fig. 11 - Experimental points of detection, UHF RFID system, for a radiated power of $100 \mathrm{~mW}$

The power level irradiated by the UHF reader antenna is programmable in the range $[100 \mathrm{~mW}$, $4 \mathrm{~W}]$. To be noted that power regulations in Europe are characterized by a limit of $500 \mathrm{~mW}$ while the maximum level of $4 \mathrm{~W}$ is permitted by US regulations. Besides the irradiated power level also the working frequency of the selected UHF reader [23] is programmable in order to support both European and US regulations. The used tags are passive devices compliant with the ISO18000-6B standard and compliant with both US and European regulations concerning frequency and power levels. The antenna is a planar one with a wide frequency working range from roughly $800 \mathrm{MHz}$ to $960 \mathrm{MHz}$.
To carry out the test campaign, the Microsoft ${ }^{\circledR}$ Visual C++ PC application controlling the UHF reader has been modified and customized in order to provide an interface suitable for testing purposes. In Fig. 12 the customized Graphical User Interface (GUI) is shown. Fig. 13 reports the maximum distance at which the tag is still detected as a function of the reader antenna power level.

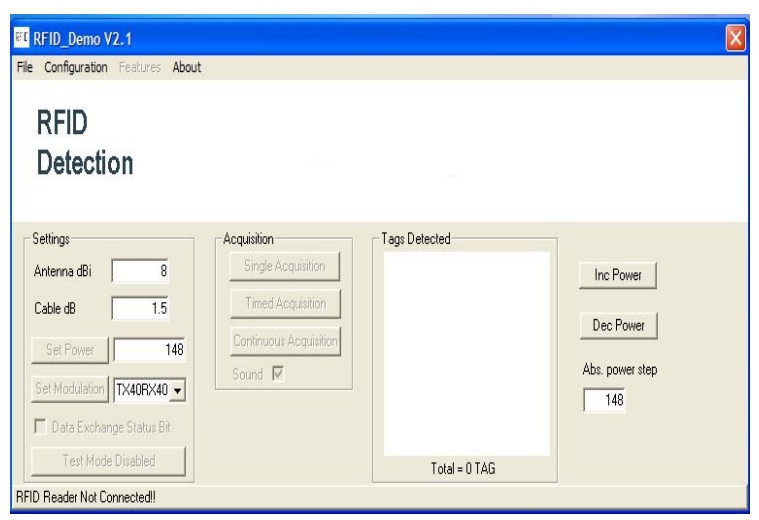

Fig. 12 - The customized GUI of the application for the UHF reader control.

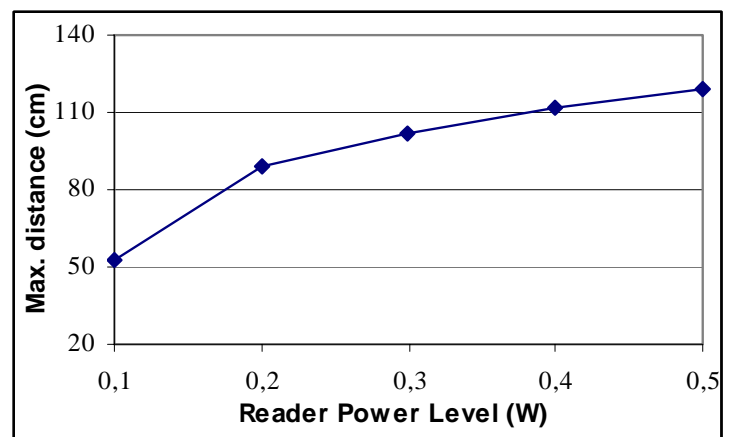

Fig. 13 - Max. distance of tag detection vs. radiated power

The experiment has been repeated with all the different stone samples interposed between the tags and the reader antenna, with and without the presence of a water level of few $\mathrm{cm}$ and with and without the presence of marble dust and mud. Differently from what measured in the case of LF and HF systems, at UHF frequencies the communication is completely shielded by water, i.e. in presence of a small layer of water, or in some cases just with a few water drops wetting the tag, the tags are not detected. These experimental results are aligned with studies [27] on water properties, see Fig. 14, proving that in the UHF range the intensity of a plane incident wave decreases to $1 / \mathrm{e}$ (that is, $63 \%$ absorbed) in a penetration distance of about 1 $\mathrm{cm}$ or lower depending on the test conditions (note that in our application the water layer can be of some $\mathrm{cm}$ ). Therefore UHF systems can not be used inside the marble machine.

The results of the test campaign applied using the configuration of Fig. 6 with different types of stones 
interposed (in a dry environment) prove that at UHF frequencies a stone sample attenuates the RF signal. Therefore the tags embedded in the conveyer belt can be detected or not depending on the distance and power level radiated by the reader.

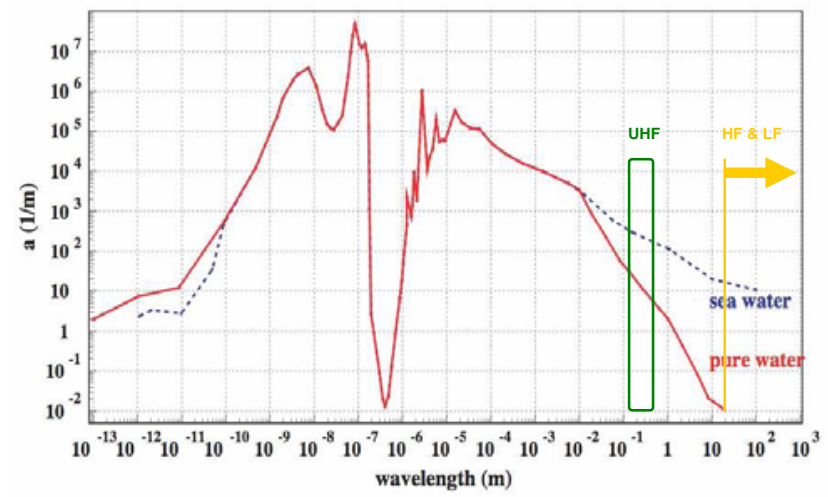

Fig. 14 - Water absorption spectrum

According to this result the detection of the marble slab with UHF systems is possible following the strategy described hereafter. First, as sketched in Fig. 6, the tags should be embedded in the conveyer belt while the reader antenna should be attached on the upper part of the machinery in a fixed position (e.g. $40 \mathrm{~cm}$ above the conveyer belt in our case study). Secondly, the emitted power level should be properly configured so that: (a) when the stone sample is interposed between the tag and the reader antenna the RF signal is attenuated under a certain bound and the tag can not be detected by the reader; (b) when the stone sample is not present the tag communicates correctly with the reader. After the test campaign in a dry environment we determined that configuring the UHF reader with a radiated power ranging from about $400 \mathrm{~mW}$ to $600 \mathrm{~mW}$ the detection of stone samples is allowed according to the above on/ off strategy. The experimental results of this test are reported in Fig. 15 for a case study of slabs sized about $30 \mathrm{~cm}$ x $30 \mathrm{~cm}$. Hence configuring the reader with a proper power level in this range (e.g. $500 \mathrm{~mW}$ ) and embedding the tags in the belt, a RFID UHF system can be used for automatic detection in the process control of marble industry. It must be pointed out that this type of detection is possible only in a dry environment, therefore it can not be used inside the machine where a water layer of several millimeters is always present.

On the contrary, outside the machine, in a dry environment, the UHF RFID system can represent an interesting alternative to traditional mechanical and optical systems to detect the presence of the marble slab at the entrance of the machine. With respect to mechanical sensors, the UHF RFID systems benefit of being contact-less. With respect to optical systems the UHF RFID solution is more robust to the presence of a dirty environment.

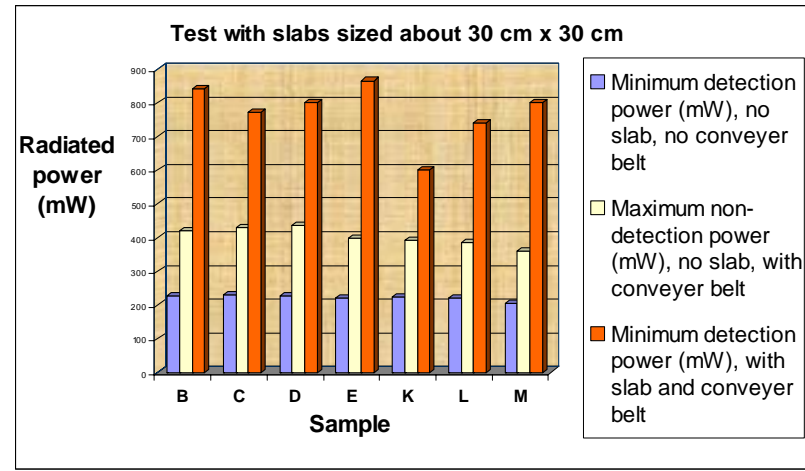

Fig. 15 - Experimental results showing the radiated power in the on/off detection strategy test

\section{EXPERIMENTAL ANALYSIS WITH MICROWAVE ACTIVE RFID SYSTEMS IN MARBLE INDUSTRY}

For the RFID UHF system at $2.45 \mathrm{GHz}$ we implemented tests similar to those carried out for the RFID system at $868 \mathrm{MHz}$. One of the main differences is represented by the fact that the 2.45 $\mathrm{GHz}$ solution, shown in Fig. 4.d, uses active tags and its maximum detection distance, without the marble slab, is up to $20 \mathrm{~m} @ 1 \mathrm{~mW}$ of effective radiated power. Since the distance to be covered in marble machine applications is below $50 \mathrm{~cm}$, during the tests we configured the $2.45 \mathrm{GHz}$ RFID tags for $1 / 16$ $\mathrm{mW}$ effective radiated power in order to limit the operating range at a few tens $\mathrm{cm}$ when the marble slab is not present. For the $2.45 \mathrm{GHz}$ active system, the slab detection strategy is not based on the reception/no reception of data packets transmitted by the active tag, but on the measure of the reception error ratio and its change in the two cases of slab interposed and not interposed between the tag and the receiver antenna. Reception errors are due to RF absorption and/or scattering by the stone. In the following, the reception ratio refers to the number of correctly received packets divided by the number of expected (i.e. transmitted) packets. For the considered 2.45 GHz RFID system, a data packet is transmitted every $250 \mathrm{~ms}$ (4 packets per second); transmission power is cyclically changed every data packet in the set of 4 different radiated power levels: $1 / 64 \mathrm{~mW}, 1 / 16 \mathrm{~mW}, 1 / 4 \mathrm{~mW}, 1 \mathrm{~mW}$. Obviously the reception error rate can be calculated as 1 minus the reception rate of correct data packets.

In order to collect experimental data, we developed an ad-hoc Matlab (from The Mathworks $^{\mathrm{TM}}$ ) application on a PC processing data received by an USB $2.45 \mathrm{GHz}$ receiver. Packets received with errors are discarded and therefore counted as non-received. The original firmware (C) on the tag and on the receiver was customized in order to format data packets according to data processing needs. 
Fig. 16 shows the reception ratio in percentage for each power level, normalized to $100 \%$, as a function of the tag-reader distance, measured in a first experiment carried out in a mixed indoor/outdoor environment. Preliminary tests were carried out in order to confirm that with transmitter and receiver placed at a short distance the reception rate was $100 \%$ for each power level, therefore excluding buffer overflow problems on the receiving PC (e.g. due to application overhead problems). For the power of 1/64 mW, Fig. 16 shows a monotonic decrease and a knee in the curve of reception ratio versus distance. Curves at different power levels have not exactly the same trend of the curve for the power of $1 / 64 \mathrm{~mW}$, probably due to reflections and/or other scattering effects in the working environment, that should of course be characterized and/or avoided in on-field applications. To achieve the best sensitivity in the reception ratio, the experimental setup for slab detection was arranged by properly displacing tag and receiver antenna so that the reception ratio without the slab was below $100 \%$, ideally on a working point in the decreasing part of the curve after the knee, in order to have a good sensitivity of the reception ratio vs. slab interposition.

During tests with the sixteen slabs we carried out about five measurement of the error ratio just a few seconds before any slab interposition in order to avoid drifts due to possible environmental effects, and then during slab interposition. The two sets of measured values are reported in Fig. 17, linked by a line identifying the progression of the experiment. With this configuration, when the marble slab is not passing, the reception ratio is from $40 \%$ to $80 \%$ while with slab interposition, in most cases, the reception ratio drops below $10 \%-20 \%$ (i.e. the reception error rate is up to $80 \%-90 \%$ ); this decrease is interpreted as detection of the stone sample, but in

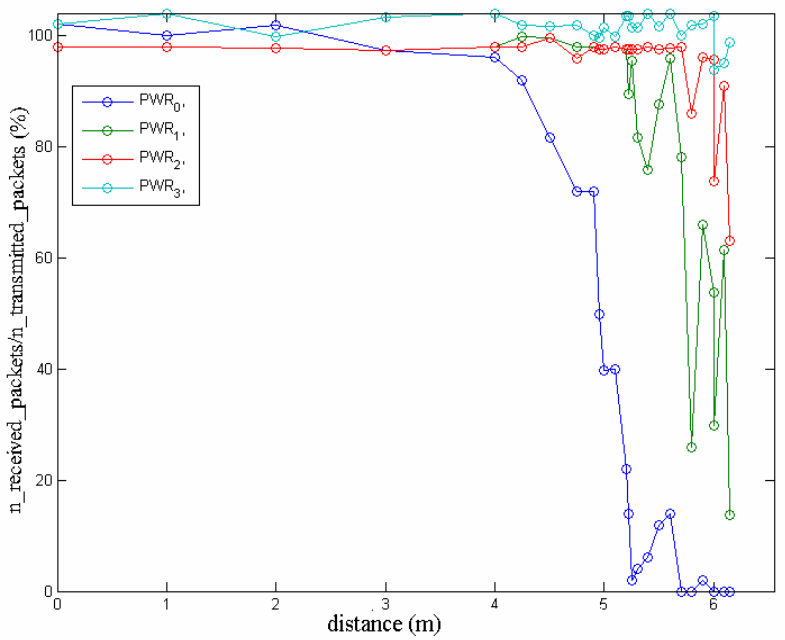

Fig. 16 - Reception ratio normalized to $100 \%$ vs. tagreader distance for emitted power levels of $1 / 64 \mathrm{~mW}$, 1/16 mW, 1/4 mW, 1 mW (PWR0, 1, 2, 3 respectively) some cases (slab B, E, J, L) the presence of the slab causes an increase of the detection ratio instead of a decrease. Since the behavior of the system depends on the specific type of stone, the described detecting technique could possibly be used only for applications involving a specific stone type but not in a machine where the type of stone sample is not known a priori.

To be noted that the considered $2.45 \mathrm{GHz}$ RFID system has two main disadvantages:

- its use is possible only at the entrance of the marble machine in a dry environment since, as in case of the $868 \mathrm{MHz}$ RFID system of Section 6, also microwave signals are absorbed by water;

- the use of active tags causes a higher cost for the tags. Therefore the solution with passive tags should be preferred for process control applications in the marble industry.

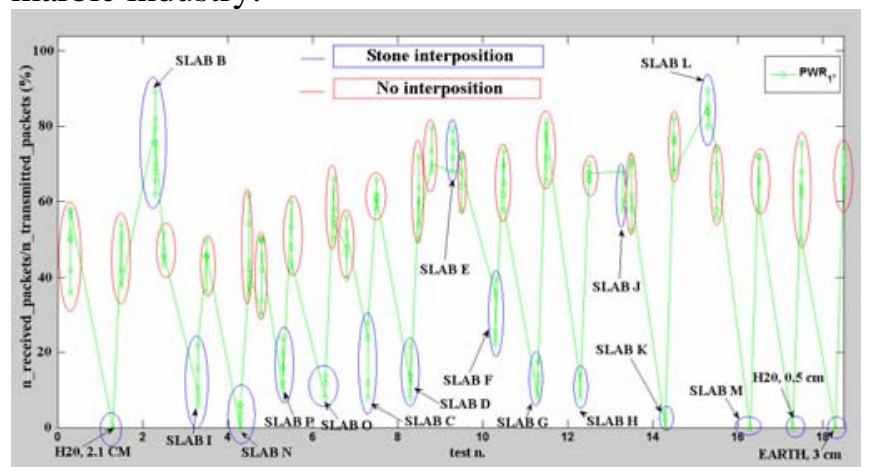

Fig. 17 - Reception ratio normalized to $100 \%$ measured with/without slab interposition for radiated power of 1/16 mW

\section{CONCLUSIONS ON EXPERIMENTAL RESULTS ABOUT RFID SYSTEMS FOR MARBLE PROCESS CONTROL}

The paper has presented an experimental analysis towards the application of 4 RFID technologies to process control in the marble industry. The final aim was the automatic detection of the presence of a marble slab under the abrasive/cutting head inside an industrial machine or outside, at the machine entrance stage. Currently used detection sensors are mechanical or optical and are used only at the entrance of the machinery: therefore the process control is missing feedback signals about the correct alignment between each marble slab and the corresponding cutting/abrasive head. Outside the machine optical sensors need frequent cleaning/ recalibration due to the dirty working environment while mechanical sensors suffer of rapid deterioration. The experimental results using a test set up reproducing the marble machine environment (with water, mud, marble dust) and considering more than 16 different types of stones proved that:

- Passive tags have to be preferred to active tags since the marble slab detection can be implemented 
with lower costs and easier system maintenance (e.g. no battery to be replaced). Table 1 summarizes the main performances of the analyzed RF detection systems using passive tags.

- All types of stones are transparent to the tested $125 \mathrm{kHz} \mathrm{LF}$ and $13.56 \mathrm{MHz}$ HF radiations; hence for these RFID systems the most suitable strategy for a reliable detection is applying the tag on the side surface of each marble slab by means of a fast dry resin. This allows the detection, even in presence of water and dirty, of the tag and of the corresponding marble slab when the tag is passing in the area covered by the reader antenna. This strategy is useful anyway to confirm to the heads controlling system the presence of the marble slab in the expected point of the machine. The tag, placed in this position, can be used also to store information for logistic applications and for the traceability of the different industrial processes applied to each slab [16].

- UHF communication at $868 \mathrm{MHz}$ and $2.45 \mathrm{GHz}$ is shielded by water and hence UHF systems can be used just outside the machine (dry environment): with respect to mechanical sensors, the RFID systems benefit of being contact-less. With respect to optical systems the RFID ones are more robust to the presence of marble dust and dirty. The detection of the marble slab is possible according to the on/off strategy described in Section 6 by properly configuring the UHF system, and possibly to the detection strategy presented in Section 7 concerning active RFIDs at $2.45 \mathrm{GHz}$. In the latter case, anyway, the behavior of the RFID system depends on the specific type of stone and therefore the described detecting technique could be suitable just for applications involving a stone type known a priori.

It is worth noting that the above described detecting techniques using passive tags require low cost tags (one for each marble slab or a set embedded in the conveyer belt) and an antenna for each head of the machine or serving a few consecutive heads. Multiple antennas (e.g. up to 4 for the RFID system in [23]) can be controlled by the same reader positioned outside the machinery since the reader and its antenna are typically connected through cables whose length amounts to some meters. Finally, future work is desirable in order to carry out the final on-field application study for integration of the RFID slab detection system in the marble machinery.

\section{ACKNOWLEDGMENT}

This work has been supported by the Tuscany Region DOCUP program in collaboration with Celver Elettronica srl. Discussions with R. Massini and A. Carrafiello are gratefully acknowledged.

Table 1. Main Performance of the RFID Systems (Passive Tags) for Marble Slab Detection

\begin{tabular}{|l|c|c|c|}
\hline \multicolumn{1}{|c|}{$\begin{array}{c}\text { RFID } \\
\text { System }\end{array}$} & $\begin{array}{c}\text { LF } \\
\mathbf{1 2 5} \mathbf{~ k H z}\end{array}$ & $\begin{array}{c}\text { HF } \\
\mathbf{1 3 . 5 6} \mathbf{~ M H z}\end{array}$ & $\begin{array}{c}\text { UHF } \\
\mathbf{8 6 8} \mathbf{~ M H z}\end{array}$ \\
\hline $\begin{array}{l}\text { Radiated } \\
\text { power }\end{array}$ & $100 \mathrm{~mW}$ & $<50 \mathrm{~mW}$ & $<500 \mathrm{~mW}$ \\
\hline $\begin{array}{l}\text { Max. stone } \\
\text { detection } \\
\text { distance }\end{array}$ & $15 \mathrm{~cm}$ & $<8 \mathrm{~cm}$ & $<40 \mathrm{~cm}$ \\
\hline $\begin{array}{l}\text { Best tag } \\
\text { placement }\end{array}$ & $\begin{array}{c}\text { Side surface of the } \\
\text { marble slab }\end{array}$ & $\begin{array}{c}\text { Embedded in } \\
\text { the conveyer } \\
\text { belt }\end{array}$ \\
\hline With water & \multicolumn{2}{|c|}{ Works } & Does not work \\
\hline $\begin{array}{l}\text { With } \\
\text { dust/dirty }\end{array}$ & \multicolumn{3}{|c}{ Works } \\
\hline
\end{tabular}

\section{REFERENCES}

[1] Barsanti Spa. Gangsaw TLD 60A/80A machine. 2009, http://www.barsantimacchine.it/

[2] Barsanti Spa. LCA200 marble polishing machine. 2008, http://www.barsantimacchine.it/

[3] D. Miorandi et al. Guest editorial: special section on wireless technologies in factory and industrial automation. IEEE Trans. on Industrial Informatics, vol. 3, n. 2, May 2007, pp.95-98.

[4] S. Saponara et al. Experimental analysis towards the application of RFID technologies in industrial marble machines”, IEEE IDAACS, Sept. 2009, Rende, Italy, pp. 67-71.

[5] Celver srl. Archimedes optical system, http://www.celver.it/new-site/prodotti/prodottiarchimedes.asp

[6] M. Bramanti et al. A Procedure to Detect Flaws inside Large Sized Marble Blocks by Ultrasound., Subsurface Sensing Tech. and Appl. Journal, n.1, vol. 2, 2001.

[7] M. A. Selver et al. Cascaded and Hierarchical Neural Networks for Classifying Surface Images of Marble Slabs, IEEE Tran. on Systems, Man and Cyb., Part C, vol. 39, 2009, pp. 426-439.

[8] J. D. Luis-Delgado et al. Classification of marble surfaces using wavelets. Electronics Letters, May 2003, vol. 39, n.9, pp. 714-715.

[9] E. Bozzi, M. Bramanti. A Planar Applicator for Measuring Surface Dielectric Constant of Materials. IEEE Tran. on Instr. and Measurement, 2000, n.4, vol. 49, pp. 773-775.

[10]D. Vaccaneo et al. Measurement system of complex permittivity of ornamental rocks in $\mathrm{L}$ frequency band. IEEE Tran. Geosc. Remote Sens., 2004, vol. 42, n. 11, pp. 2490-2498.

[11]S. Coker et al. In-process control of surface roughness due to tool wear using a new ultra- 
sonic system. Journal of Machine Tools and Manufacture, 1996, vol. 43, n. 3, pp. 411-422.

[12] S. Saponara et al. Capacitive sensing for contact-less proximity detection in industrial marble machines. Sensors and Transducers, 2010, vol. 8, pp. 26-41.

[13] B. Osoincah. Proximity Capacitive Sensor Technology for Touch Sensing Applications. Freescale White Paper, 2007, pp. 12.

[14] I. Ar, Y. S. Akgul. A generic system for the classification of marble tiles using Gabor filters. IEEE ISCIS'08, pp. 1-6.

[15] J. Martinez-Alajarin et al. Automatic system for quality-based classification of marble textures. IEEE Trans. on Systems, Man, and Cybernetics, Part C, 2005, vol. 35, pp. 488-497.

[16] RFIDline. RFid project: Special technology on marble and granite”, 2007, http://www. rfidstone.com

[17] R. Wessel. Italian stone supplier uses RFID to track marble, granite. RFIDJournal, 2007.

[18] K. Kwon et al. Intelligent process control system with RFID cuboid. IEEE Int. Conf. on Electr. Commerce, Taipei, Taiwan, 2009, pp. 18.

[19] G. Fenu. RFID-based supply chain traceability system. IEEE IECON 2009, pp.2672-2677.

[20] Byoung-Suk Choi et al. An improved localization system with RFID technology for a mobile robot. IEEE IECON, 2008, pp. 34093413.

[21] EM Microelectronic. EM4102 Read only contact less identification device. 2005.

[22] PSION Teklogic. Workabout Pro guide. 2004.

[23] CAEN SpA. A928 long range UHF reader data sheet. 2007, http://www.caen.it/rfid/

[24] Open $2.4 \quad \mathrm{GHz}$ RFID Sputnik. http://www.openbeacon.org/

[25] Y.-C. Choi et al. A Multi Standard 13.56 MHz RFID Reader System. Proc. of ITC-CSCC 2008, pp. 1073-1076.

[26] N. Choi et al. Design of a $13.56 \mathrm{MHz}$ RFID System. ICACT 2006, pp. 840-843.

[27] M. Chaplin. Water structure and science; water and microwaves. http://www.lsbu.ac.uk/water/.

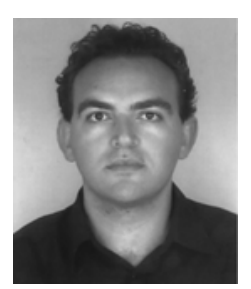

Sergio Saponara got the Master of Science degree, cum laude, and the Ph.D. in Electronic Engineering from the University of Pisa in 1999 and 2003 respectively. In 2002 he was with IMEC, Leuven (B), as Marie Curie Research Fellow. He is senior researcher at University of Pisa in the field of electronic circuits and systems for telecom, multimedia, space and automotive. He holds the chair of electronic systems for automotive and automation at the Faculty of Engineering. He coauthored 110 scientific publications and 5 patents.

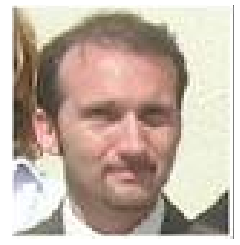

Fabrizio lacopetti got the Master of Science in Electronic Engineering from University of Pisa in 2005. From 2005 to 2008 he has been a research contractor of the University of Pisa where he

is currently a staff technical engineer at the Department of Information Engineering. His main research interests include microcontroller-based systems for industrial automation and biomedical applications. He co-authored 10 scientific publications and he holds 2 patents.

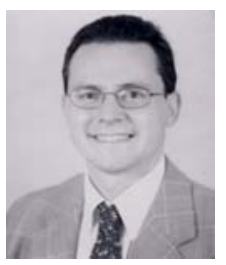

Luca Fanucci got the Master of Science and the Ph.D. degrees in Electronic Engineering from University of Pisa in 1992 and 1996, respectively. From 1992 to 1996, he was with ESA/ESTEC, Noordwijk (NL), as research fel-

low.From 1996 to 2004 he was a senior researcher of the CNR in Pisa. He is Professor of Microelectronics at the University of Pisa. His research interests include VLSI architectures for integrated circuits and systems. Prof. Fanucci coauthored more than 150 scientific publications and he holds more than 10 patents. He was program chair of Euromicro DSD 2008 and DATE Designer's Forum.

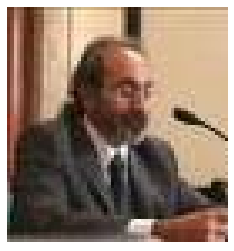

Bruno Neri got the Master of Science degree, cum laude, from the University of Pisa in 1980. From 1983 he has been first Assistant Professor, then Associate Professor and since

2000 Full Professor of Electronics for Radiofrequencies (RF) and Instrumentation at University of Pisa. He is the Director of the Department of Information Engineering. His research interests focused on failure mechanisms of microelectronic devices, on extremely low-noise instrumentation, and currently on RF/microwave circuits and wireless systems. He has been the leading researcher for several EU and national projects on the above themes. He co-authored more than 100 scientific publications and two patents. 